\title{
The impact of infertility diagnosis on embryo-endometrial dialogue
}

\author{
Jason C Parks ${ }^{1,2}$, Blair R McCallie ${ }^{1,2}$, Alyssa L Patton ${ }^{1}$, Zain A Al-Safi ${ }^{3}$, Alex J Polotsky ${ }^{3}$, \\ Darren K Griffin ${ }^{2}$, William B Schoolcraft ${ }^{1}$ and Mandy G Katz-Jaffe ${ }^{1}$ \\ ${ }^{1}$ Colorado Center for Reproductive Medicine, Lone Tree, Colorado, USA, ${ }^{2}$ University of Kent, Canterbury, UK and \\ ${ }^{3}$ Department of Obstetrics and Gynecology, University of Colorado School of Medicine, Aurora, Colorado, USA
}

Correspondence should be addressed to J C Parks; Email: jparks@flcolo.com

\begin{abstract}
Initial stages of implantation involve bi-directional molecular crosstalk between the blastocyst and endometrium. This study investigated an association between infertility etiologies, specifically advanced maternal age (AMA) and endometriosis, on the embryo-endometrial molecular dialogue prior to implantation. Co-culture experiments were performed with endometrial epithelial cells (EEC) and cryopreserved day 5 blastocysts $(n=41 \geq$ Grade $3 \mathrm{BB})$ donated from patients presenting with AMA or endometriosis, compared to fertile donor oocyte controls. Extracellular vesicles isolated from co-culture supernatant were analyzed for miRNA expression and revealed significant alterations correlating to AMA or endometriosis. Specifically, AMA resulted in 16 miRNAs with increased expression $(P \leq 0.05)$ and strong evidence for negative regulation toward 206 target genes. VEGFA, a known activator of cell adhesion, displayed decreased expression $(P \leq 0.05)$, validating negative regulation by 4 of these increased miRNAs: miR-126; 150; 29a; 29b $(P \leq 0.05)$. In endometriosis patients, a total of 10 significantly altered miRNAs displayed increased expression compared to controls (miR-7b; 9; 24; 34b; 106a; 191; 200b; 200c; 342-3p; 484) $(P \leq 0.05)$, targeting 1014 strong evidence-based genes. Three target genes of miR-106a (CDKN1A, E2F1 and RUNX1) were independently validated. Functional annotation analysis of miRNA-target genes revealed enriched pathways for both infertility etiologies, including disrupted cell cycle regulation and proliferation $(P \leq 0.05)$. These extracellular vesicle-bound secreted miRNAs are key transcriptional regulators in embryo-endometrial dialogue and may be prospective biomarkers of implantation success. One of the limitations of this study is that it was a stimulated, in vitro model and therefore may not accurately reflect the in-vivo environment.

Reproduction (2018) 155 543-552
\end{abstract}

\section{Introduction}

The World Health Organization estimates that $10 \%$ of couples worldwide will struggle with infertility. Although a small number of these reproductive-age couples may succeed using conventional methods over many months and possibly years, most will require the use of assisted reproductive technologies (ARTs) and often in vitro fertilization (IVF) to establish a pregnancy (Thoma et al. 2013). Although considered relatively successful, $70 \%$ of IVF cycles will not result in a live birth (Weimar et al. 2013).

An intricate, bi-directional molecular dialogue between embryo and endometrium during the window of implantation, approximately 6-12 days post ovulation, is crucial for success (Wilcox et al. 1999). Without a precise interchange between the two, implantation will ultimately fail. Key genes involved with cell cycle regulation, as well as ion-binding and signal-transporting proteins, have been identified as contributors to the molecular dialogue between the embryo and a receptive endometrium by sampling the uterine microenvironment (Hannan et al. 2012, RuizAlonso et al. 2012). MicroRNAs (miRNAs) also play an important role during the window of implantation and are expressed by both the implanting embryo and the receptive endometrium (Blakaj \& Lin 2008, Laurent 2008). Altered expression of miRNAs (including miR30b, miR-30d and miR-494) have been shown to regulate endometrial receptivity (Altmae et al. 2013).

An in vitro co-culture system can provide valuable insight into the initial bi-directional dialogue (Simon et al. 1998). In vitro studies utilizing an embryo/ endometrial co-culture system have observed key components of the molecular crosstalk between the embryo and endometrial epithelial cells (EEC). However, limited information regarding this interaction is known (Barmat et al. 1999, Simon et al. 1999, Rubio et al. 2000).

Our unique study utilized a human uterine luminal endometrial epithelial cell and human blastocyst co-culture system to investigate the molecular dialogue at the time of adhesion, in association with two major causes of female factor infertility (endometriosis and advanced maternal 
age). Advanced maternal age (AMA) is associated with a substantial linear decline in reproductive potential and is the major cause of female infertility (Rosenwaks et al. 1995). There are many factors contributing to the significant decrease in live births as women approach their 5th decade, including diminishing ovarian reserve (Zhang 2015), and the increase in oocyte chromosomal aneuploidy (Harton et al. 2013). Aneuploidy screening in IVF cycles has allowed for the transfer of euploid blastocysts, resulting in higher implantation rates, independent of maternal age (Schoolcraft et al. 2011). However, there are other variables beyond the oocyte/embryo chromosome constitution that impact the ability of a euploid blastocyst to successfully implant. Traditionally, AMA patients are shown to have a longer infertility duration and diminished ovarian reserve and require a higher dose of gonadotropins in controlled ovarian hyperstimulation, all resulting in poorer oocyte quality (Ocal et al. 2012).

Another pathological cause of infertility, widely diagnosed and treated within the field of reproductive medicine, is endometriosis. Endometriosis is a disease which causes tissue normally found within the uterus, to migrate and grow outside of it (Buck Louis et al. 2011). This debilitating disease is often painful, impacting a woman's quality of life, and greatly reduces the chances of conception. It is estimated that nearly half of all women with this diagnosis will have difficulty achieving a pregnancy (Practice Committee of the American Society for Reproductive Medicine 2006, Ozkan et al. 2008). The causes of endometriosis remain unclear; however a genetic predisposition is suspected as a common risk factor, including a family history of the condition (Wenzl et al. 2003). Several pathways have also been identified within patients diagnosed with endometriosis which may contribute to the poor oocyte quality associated with the disease (Shebl et al. 2017). Within infertile populations, it is estimated that the prevalence of patients diagnosed with endometriosis is approximately $26.13 \%$ in women undergoing laparoscopy (GarciaVelasco \& Quea 2005, Rizk et al. 2015).

The aim of this study is to investigate these different primary infertility etiologies within a controlled co-culture system, to elucidate differences within the secretome. We found significant differences in the miRNA content within the extracellular vesicles of the supernatant, as well as differences in gene expression within the co-cultured endometrial cells and blastocysts which may impact endometrial function during the window of implantation.

\section{Materials and methods}

\section{Embryos}

Surplus, cryopreserved, hatching, transfer-grade blastocysts as identified by the Gardner \& Schoolcraft grading system (Gardner \& Schoolcraft 1999) as $\geq$ Grade 3BB on day 5 of embryonic development $(n=41)$ were donated with patient consent and IRB approval. Blastocysts were derived from IVF cycles: young fertile oocyte donor controls (fertile control group) with no history of female or male factor infertility, based on WHO guidelines (maternal age $\leq 32$ years old, BMI $<29$, non-smoker, non-drug user, with normal ovarian reserve and regular menstrual cycles, $n=15$ ), infertile women of AMA group without pre-implantation genetic diagnosis ( $\geq 39$ years or older, with no other infertility diagnosis, $n=14$ ), and younger female patients diagnosed with endometriosis as the only cause of infertility (mean maternal age of 34.3, with 11 of the 12 patients $\leq 33$ years old, $n=12$ ). Subjects underwent an ovarian stimulation protocol based on clinical discretion which included gonadotropin-releasing hormone $(\mathrm{GnRH})$ agonist down-regulation, microdose $\mathrm{GnRH}$ agonist flare or $\mathrm{GnRH}$ antagonist with Menopur (Ferring Pharmaceuticals, Saint-Prex, Switzerland) or Bravelle (Ferring Pharmaceuticals) with or without clomiphene citrate. Final oocyte maturation trigger was induced when the lead follicle reached $20 \mathrm{~mm}$ mean diameter using an intramuscular injection of hCG, a subcutaneous injection of leuprolide acetate, or a combination of both, based on clinical judgment. Routine oocyte retrieval was performed transvaginally under ultrasound guidance $35 \mathrm{~h}$ post trigger. Patients within the endometriosis group were asymptomatic at the time of their oocyte retrieval and biopsy. Patients were excluded from the study if they had any other infertility comorbidities including polycystic ovary syndrome and depleted ovarian reserve. Embryos were vitrified and warmed according to routine laboratory procedures described previously (Kuwayama et al. 2005). Total time spent frozen varied, with an average time of approximately 4 years.

\section{Endometrial biopsies}

Endometrial biopsies were collected from either young, fertile oocyte donors (fertile control group, $n=7$ ) or from patients diagnosed with endometriosis who were currently asymptomatic at the time of their oocyte retrieval $(n=2)$. Subjects were stimulated as described earlier. Endometrial biopsies measured $1.5-3.0 \mathrm{~cm}$ in length and approximately $0.5 \mathrm{~cm}$ in diameter. For every endometrial biopsy collected, tissue was minced into $<1 \mathrm{~mm}$ sections and incubated at $4^{\circ} \mathrm{C}$ overnight in a $0.1 \%$ collagenase solution (Sigma-Aldrich). The primary epithelial endometrial cells (EEC) were isolated by vigorously vortexing and rinsing the cell milieu in $10 \mathrm{~mL}$ of room temperature DMEM (Sigma-Aldrich), prior to resting the cells for 5-10 min as they settled into distinct layers of EEC and stromal cells. The supernatant containing stromal cells was then removed and the process was repeated three times until no further stromal cells remained. $500 \mu \mathrm{L}$ of the EEC/DMEM was then added to 6 wells of a Falcon Multiwell 24-well Tissue Culture Plate (Corning Incorporated) (Mercader et al. 2003). Cells were cultured in a media composed of $75 \%$ Dulbecco Modified Eagle Medium (Sigma) and 25\% MCDB105 (Sigma) containing antibiotics, $5 \mu \mathrm{g} / \mathrm{mL}$ insulin (Sigma) and supplemented with $10 \%$ exosome-depleted fetal bovine serum (System Biosciences, Palo Alto, CA, USA) and observed daily to monitor the development of an EEC monolayer. The monolayer reached acceptable levels of well coverage at 
$>65 \%$ after $4-6$ days. The growth medium was then replaced with a serum-free medium for embryo development and supplemented with $4 \%$ recombinant human serum albumin (Vitrolife, Englewood, CO, USA). For each endometrial biopsy processed, 4-6 wells received an individual thawed blastocyst which was co-cultured for $48 \mathrm{~h}$ at $5 \% \mathrm{O}_{2}$ and $6 \% \mathrm{CO}_{2}$ at $37^{\circ} \mathrm{C}$ based on current laboratory practices for human blastocyst culture (Kovacic 2012).

\section{Co-culture collection}

Blastocysts were co-cultured together for $48 \mathrm{~h}$ prior to collection. AMA or fertile control donor blastocysts were co-cultured with fertile control donor endometrial tissue. Additionally, the endometriosis subjects were divided into three groups: fertile control donor blastocysts co-cultured with fertile control donor endometrium (CD), fertile control donor blastocysts co-cultured with endometrial cells from patients diagnosed with endometriosis (ED) and blastocysts and endometrial cells that were both obtained from patients diagnosed with endometriosis (EE). Adhesion by the blastocyst to the endometrial monolayer was observed twice in the AMA group, twice in the fertile control group and once in both of the endometriosis groups. Adhered blastocysts were gently scraped off the monolayer prior to collection. Following co-culture, blastocysts were graded and rinsed in $10 \%$ phosphate buffered saline-bovine serum albumin (PBSBSA) before lysis in $10 \mu \mathrm{L}$ of extraction buffer (PicoPureRNA Isolation Kit, Thermo Fisher Scientific). Co-culture supernatant was collected $(500 \mu \mathrm{L})$ and snap-frozen in liquid nitrogen. The monolayer was visually measured for $100 \%$ confluence, then rinsed with DPBS (Thermo Fisher) and incubated at $37^{\circ} \mathrm{C}$ with $1 \times$ diluted TrypLESelect $(10 \times)$ (Thermo Fisher) until cells lifted from the bottom of the plate $(5-15 \mathrm{~min})$. Each well of EEC were collected individually. The pelleted EEC were lysed with $50 \mu \mathrm{L}$ Extraction Buffer (PicoPureRNA Isolation Kit, Thermo Fisher) and snap-frozen with liquid nitrogen. All samples were stored at $-80^{\circ} \mathrm{C}$ until further analysis (Fig. 1 ).

\section{Supernatant extracellular vesicle miRNA analysis}

Extracellular vesicles were isolated using Total Exosome Isolation Reagent (from cell culture media) (Thermo Fisher). $350 \mu \mathrm{L}$ of supernatant was combined with $175 \mu \mathrm{L}$ of reagent and vortex-mixed prior to an overnight incubation at $4{ }^{\circ} \mathrm{C}$. Samples were centrifuged under refrigerated conditions for $1 \mathrm{~h}$, then the supernatant was aspirated and discarded. The resulting pellet was lysed in $10 \mu \mathrm{L}$ of lysis solution containing DNase (Taqman MicroRNA Cells-to- $C_{T}$ Kit, Thermo Fisher) and incubated at room temperature for $8 \mathrm{~min}$. Lysed miRNA was reverse-transcribed using the Taqman MicroRNA Reverse Transcription Kit with MegaPlex RT Human Primer Pool A (Thermo Fisher) in a final volume of $7.5 \mu \mathrm{L}$ under the following thermal cycling conditions: 40 cycles at $16^{\circ} \mathrm{C}$ for $2 \mathrm{~min}, 42^{\circ} \mathrm{C}$ for $1 \mathrm{~min}$ and $50^{\circ} \mathrm{C}$ for $1 \mathrm{~s}$ followed by a hold for $5 \mathrm{~min}$ at $85^{\circ} \mathrm{C}$. cDNA was pre-amplified using the Taqman PreAmp Mastermix with MegaPlex PreAmp Human Primer Pool A (Thermo Fisher). $100 \mu \mathrm{L}$ of the diluted pre-amplified product was added to each well of the Taqman Human MicroRNA Array

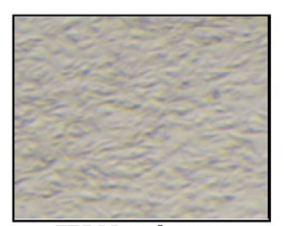

EEC Monolayer
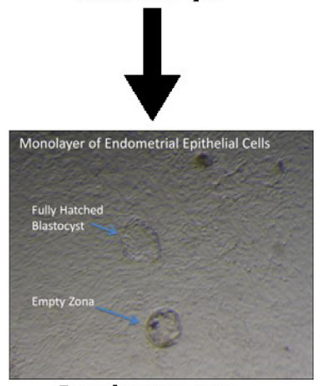

Co-culture system
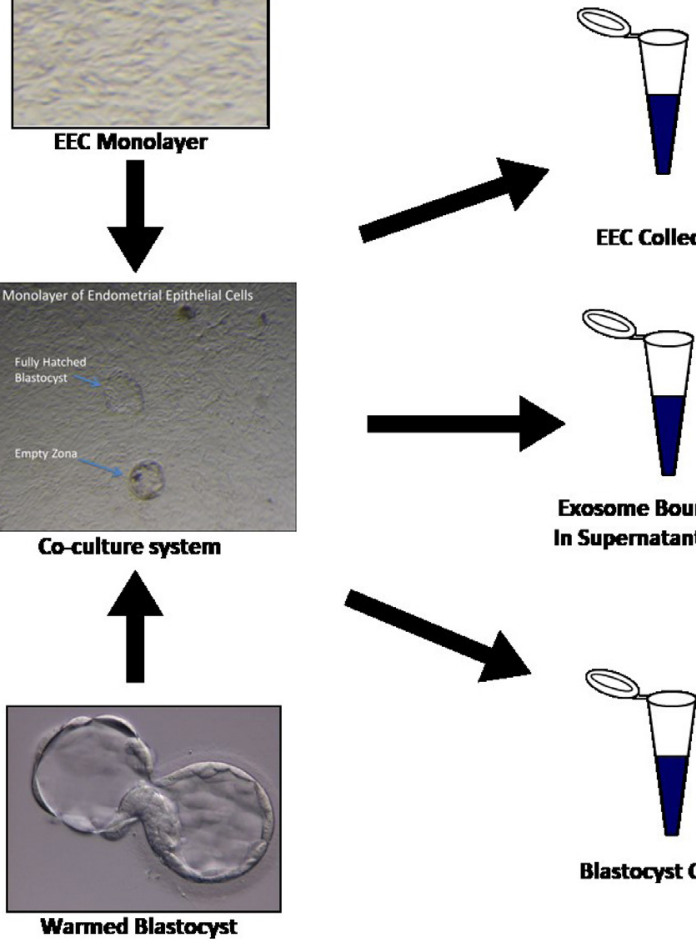

EEC Collection

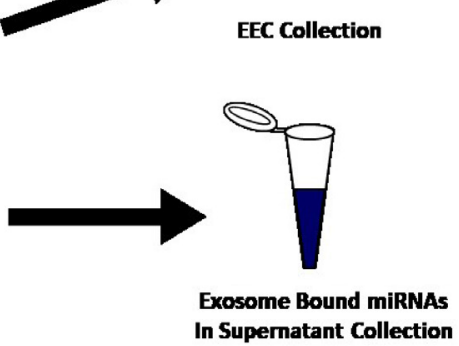

Figure 1 Experimental design for embryo co-culture with cultured endometrial cells.

Card A and was then run on the 7900 HT Fast Real-Time PCR System (Thermo Fisher) under the following thermal cycling conditions: $50^{\circ} \mathrm{C}$ for $2 \mathrm{~min}, 94.5^{\circ} \mathrm{C}$ for $10 \mathrm{~min}$ and 40 cycles at $97^{\circ} \mathrm{C}$ for $30 \mathrm{~s}$ and $59.7^{\circ} \mathrm{C}$ for $1 \mathrm{~min}$. Data were analyzed using the RQ Manager 1.2.1 (Thermo Fisher), and statistical analysis was performed using REST 2009 software (Qiagen). miRNAs that displayed significantly altered expression profiles were further investigated for target gene analysis using miRTarBase (http://mirtarbase.mbc.nctu.edu.tw/index.php), a database of experimentally validated miRNA-target interactions that are classified as strong evidence based if there is sufficient published empirical evidence.

\section{Blastocyst gene expression}

Individual blastocysts stored in $10 \mu \mathrm{L}$ of extraction buffer (PicoPure RNA Isolation Kit, Thermo Fisher) were incubated at $42^{\circ} \mathrm{C}$ for $30 \mathrm{~min}$ prior to isolating RNA. This procedure entailed adding 1 volume of $70 \% \mathrm{EtOH}$ to each sample before transferring to a pre-conditioned purification column. Samples were then DNase-treated using the RNase-Free DNase Set (Qiagen) and eluted in $20 \mu \mathrm{L}$ following several washes. All $20 \mu \mathrm{L}$ of purified total RNA was reverse-transcribed using the High-Capacity cDNA Reverse Transcription Kit (Thermo Fisher) in a final volume of $40 \mu \mathrm{L}$. The reaction occurred in a thermal cycler at $25^{\circ} \mathrm{C}$ for $10 \mathrm{~min}$ followed by $37^{\circ} \mathrm{C}$ for $2 \mathrm{~h}$. cDNA samples were diluted 1:5 with nuclease-free water and a $5 \mu \mathrm{L}$ template was used for real-time PCR using the 
Power SYBR Green PCR Master Mix (Thermo Fisher) and a $5 \mu \mathrm{M}$ primer mix (forward+reverse) in a $25 \mu \mathrm{L}$ final volume. Duplicates of each sample were performed on the ABI 7300 Real-Time PCR System under the following thermal cycling conditions: $95^{\circ} \mathrm{C}$ for $10 \mathrm{~min}, 40$ cycles at $95^{\circ} \mathrm{C}$ for $15 \mathrm{~s}$ and $60^{\circ} \mathrm{C}$ for $1 \mathrm{~min}$, followed by a dissociation stage to determine melt temperatures. Standard curves were also employed for each gene using Universal Human Reference RNA (Agilent) in 10-fold serial dilutions and an internal housekeeping gene (PPIA) was used to normalize each sample.

\section{Endometrial cell molecular analysis}

EEC samples were lysed in $75 \mu \mathrm{L}$ of lysis solution and miRNAs were isolated using the RNaqueous-Micro Kit (Thermo Fisher) which required the addition of 1.25 volumes of $100 \% \mathrm{EtOH}$ prior to loading onto a micro filter cartridge assembly. Samples were DNase-treated in the same manner as the blastocyst samples, and washed several times before eluting in $10 \mu \mathrm{L}$ of elution solution previously heated to $75^{\circ} \mathrm{C}$ (performed twice for maximum recovery, $20 \mu \mathrm{L}$ final volume). miRNA samples were then concentrated down to a $10 \mu \mathrm{L}$ volume using a vacuum concentrator (Vacufuge Plus, Eppendorf).

miRNA samples were pre-amplified, diluted and evaluated using the Taqman Human MicroRNA Array Card A (Thermo Fisher) as described in the earlier supernatant exosome miRNA analysis section. Data were analyzed using the RQ Manager 1.2.1 (Thermo Fisher) and statistical analysis was performed as described in the following.

\section{Statistical analysis}

A Mann-Whitney $U$ test was used to compare differences in the age of the donor women (fertile control, AMA and endometriosis blastocysts), with significance at $P \leq 0.05$. The PCR reaction efficiencies recorded $R^{2}$ values $\geq 0.9$ and correlation coefficients were calculated to be $>0.99$. Statistical analysis was performed with REST-2009 software (Qiagen) which uses bootstrap randomization techniques to correct for exact PCR efficiencies with mean crossing point deviations between sample and control groups to determine an expression ratio that is tested for significance by a pair wise fixed reallocation randomization test. The standard error and the $95 \%$ confidence interval $(\mathrm{Cl})$ given by REST-2009 software are not derived from Ct values. They are the standard error and the $\mathrm{Cl}$ for the 50,000 iterations the program runs to calculate the $P$ value; thus, no error bars are generated for graphical presentation. Gene expression fold differences with $P \leq 0.05$ were considered statistically significant.

\section{Results}

\section{Co-culture of AMA blastocysts and fertile control blastocysts}

The age of the donors in the fertile control blastocyst group were significantly different than those in the AMA blastocyst group $(P \leq 0.05)$. Isolated cargo of extracellular vesicles from supernatant collected after co-culture of fertile endometrial epithelial cells with blastocysts from infertile AMA patients revealed altered miRNA expression profiles compared to fertile control blastocysts. A total of 16 miRNAs showed increased expression and 2 exhibited reduced expression in association with the maternal age of the blastocyst relative to the internal housekeeping gene $R N U 48$ ( $P \leq 0.05$, Fig. 2). Target gene investigation of these 18 altered miRNAs using miRTarBase (http://mirtarbase. mbc.nctu.edu.tw/index.php) revealed 576 genes, 206 of which displayed strong evidence as defined by Western blot, qPCR or reporter assay as described previously. Of these strong evidence-based genes, VEGFA was identified as a target gene, specifically regulated by four of the altered miRNAs: miR-126, 150, 29a and 29b. Examination of VEGFA transcription by qPCR observed decreased expression in both the corresponding endometrial epithelial cells and blastocysts from infertile AMA patients following co-culture, specifically a $50 \%$ fold change reduction compared to fertile controls ( $P \leq 0.05$; Fig. 3$)$.

\section{Co-culture of endometriosis blastocysts or fertile control blastocysts on donor endometrium or endometriosis endometrium}

The ages of the donors in the fertile control blastocyst group were not significantly different from those in the endometriosis blastocyst group ( $P \geq 0.05$; ns). Isolated extracellular vesicles from supernatant collected after

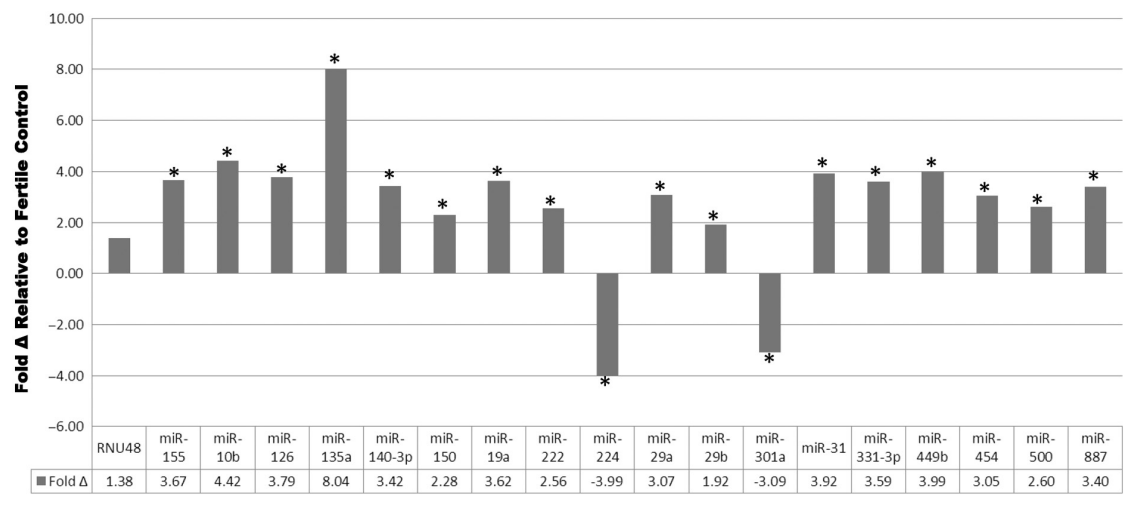

Figure 2 Differential expression of extracellular vesicle-bound miRNAs in co-culture supernatant in association with maternal age $\left({ }^{*} P \leq 0.05\right)$. 


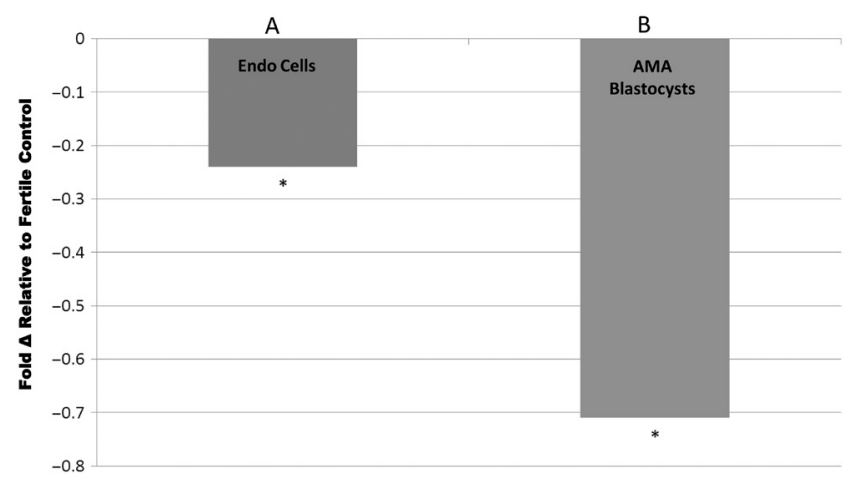

Figure 3 Target gene VEGFA expression in endometrial cells (A) and AMA blastocysts (B) compared to young fertile controls $(* P \leq 0.05)$.

co-culture of endometriosis EEC with blastocysts from endometriosis patients revealed an altered miRNAome compared to fertile controls. Specifically, 10 miRNAs showed significant decreased expression including miR-7b miR-24, miR-34b, miR-200c, miR342-3p, miR-9, miR-484, miR-200b, miR-106a and miR-191 ( $P \leq 0.05$; Figs 4 and 5) compared to fertile control endometrium co-cultured with fertile control blastocysts. Using mirTarBase, strong evidence-based target genes were revealed, including, among others, 27 target genes for miR-106a. Of these 27 target genes CDKN1A, E2F1 and RUNX1 were chosen for further investigation because of their known roles in association with implantation in murine (Cdkn1a) (Das 2009) and human models (E2F1 and RUNX1) (Tapia et al. 2011, Tapia-Pizarro et al. 2014). qPCR analysis observed increased expression of CDKN1A in endometriosis blastocysts compared to fertile controls when co-cultured with fertile control derived EEC $(P \leq 0.05)$. This expression was further increased when endometriosis derived blastocysts were co-cultured with endometriosis derived EEC ( $P \leq 0.05$; Fig. 6A). Endometriosis EEC co-cultured with fertile control blastocysts displayed increased expression of E2F1 and RUNX1 $(P \leq 0.05)$. This significant difference in expression was further increased when endometriosis derived blastocysts were co-cultured using endometriosis derived $\mathrm{EEC}(P \leq 0.05$; Fig. 6B).

\section{Gene ontology and pathway analysis}

DAVID Bioinformatics Resources 6.8 (https://david. ncifcrf.gov/) was utilized for functional annotation clustering analyses of differentially expressed miRNAs to explore the GO biological processes and pathways, common to both infertility etiologies, and included cellular development, proliferation and cell cycle regulation.

The enriched pathways and processes that were identified as being specific to only the AMA etiology included PI3K-Akt and vascular endothelial growth factor (VEGF) signaling, as well as biological processes involving cellular development and migration, adhesion and cell cycle regulation $(P \leq 0.05$; Supplementary Table 1, see section on supplementary data given at the end of this article).

For the endometriosis etiology group, enriched biological processes and pathways crucial for development and implantation were revealed within functional annotation clusters of the 1014 strong evidencebased target genes, including positive regulation of cell proliferation, G1/S transition of mitotic cell cycle and the p53 signaling pathway and angiogenesis $(P \leq 0.05)$. Additionally, cell cycle regulation and arrest, positive regulation of cell proliferation, negative regulation of apoptotic processes and negative regulation of G1/S transition of mitotic cell cycles were also identified (Supplementary Table 2).

\section{Discussion}

This is a novel study investigating the bi-directional molecular communication at the time of implantation in association with infertility etiologies. Blastocysts from patients presenting with AMA or endometriosis were

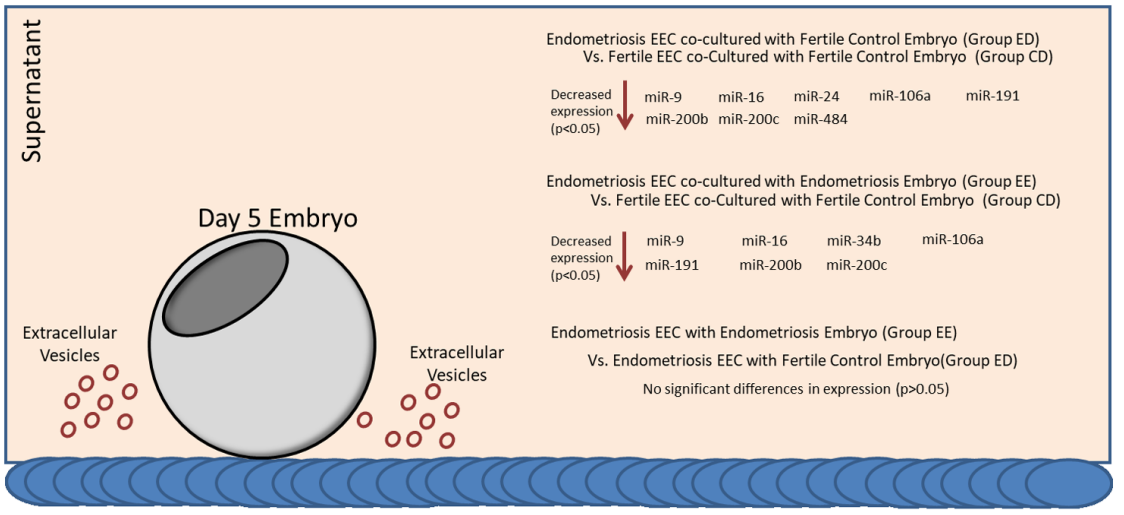

EEC Monolayer
Figure 4 Experimental groups $(C D=$ fertile control endometrium/fertile control blastocyst; $\mathrm{ED}=$ endometriosis endometrium/fertile control blastocyst; $\mathrm{EE}=$ endometriosis endometrium/endometriosis blastocyst) and the affected miRNAs for embryos and endometrial cells derived from patients diagnosed with endometriosis compared with fertile control. 


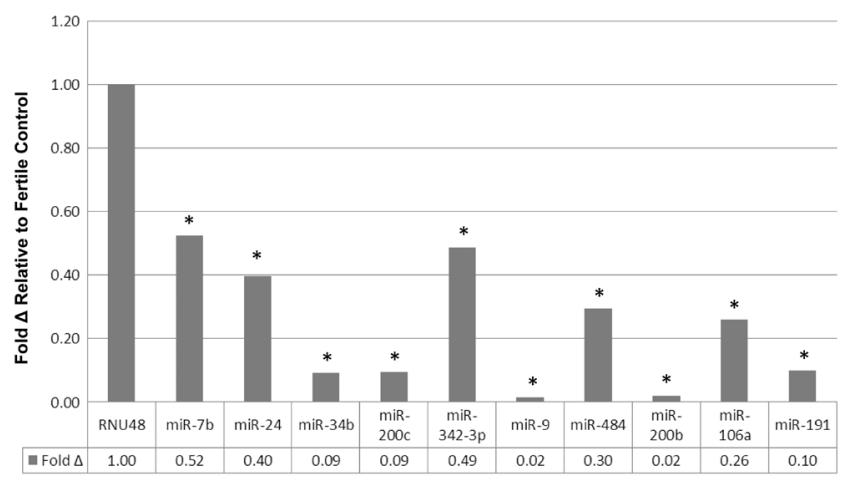

Figure 5 Extracellular vesicle-bound microRNA expression of co-culture supernatant. Endometriosis derived blastocysts cultured on a monolayer of endometriosis derived endometrial tissue and fertile control derived endometrial tissue, fertile control oocyte derived blastocysts $\left({ }^{*} P \leq 0.05\right)$.

co-cultured on a monolayer of endometrial cells to capture the molecular dialogue between a blastocyst and the endometrium during the initial stages of implantation. Specific extracellular vesicle-bound miRNAs derived from co-culture supernatant were identified to have significantly altered expression in association with AMA or endometriosis, highlighted by the differences within embryo-endometrial communication depending on the source of the embryo or endometrium. This impacted downstream target gene expression in either blastocysts or endometrial cells during the window of implantation.

\section{Advanced maternal age}

It is well documented that infertile women presenting with advanced maternal age have some of the poorest prognoses with regard to oocyte and blastocyst quality. Additionally, there is some evidence that the endometrium is also affected by advanced age, resulting in embryo-endometrial asynchrony (Shapiro et al. 2016). The molecular dialogue between the embryo and endometrium is crucial for successful implantation, and it is believed that miRNA-bound extracellular vesicles are involved in this bi-directional molecular communication.

A total of 18 extracellular vesicle-bound miRNAs displayed altered expression with the presence of AMA blastocysts. One example was miR-150, a critically important miRNA for lineage cell differentiation, which also plays a role in mediating an appropriate inflammatory response within the endometrial environment. This response includes not only the endometrial epithelial cells and stromal cells, but also the immune-related cells and vasculature (Pan \& Chegini 2008, Elton et al. 2013, Goossens et al. 2013). Pregnancy is a pro-inflammatory state and molecular miscommunication may result in an improperly coordinated auto-immune response, leading to inflammation at the implantation site, causing implantation failure and embryonic death. This is a
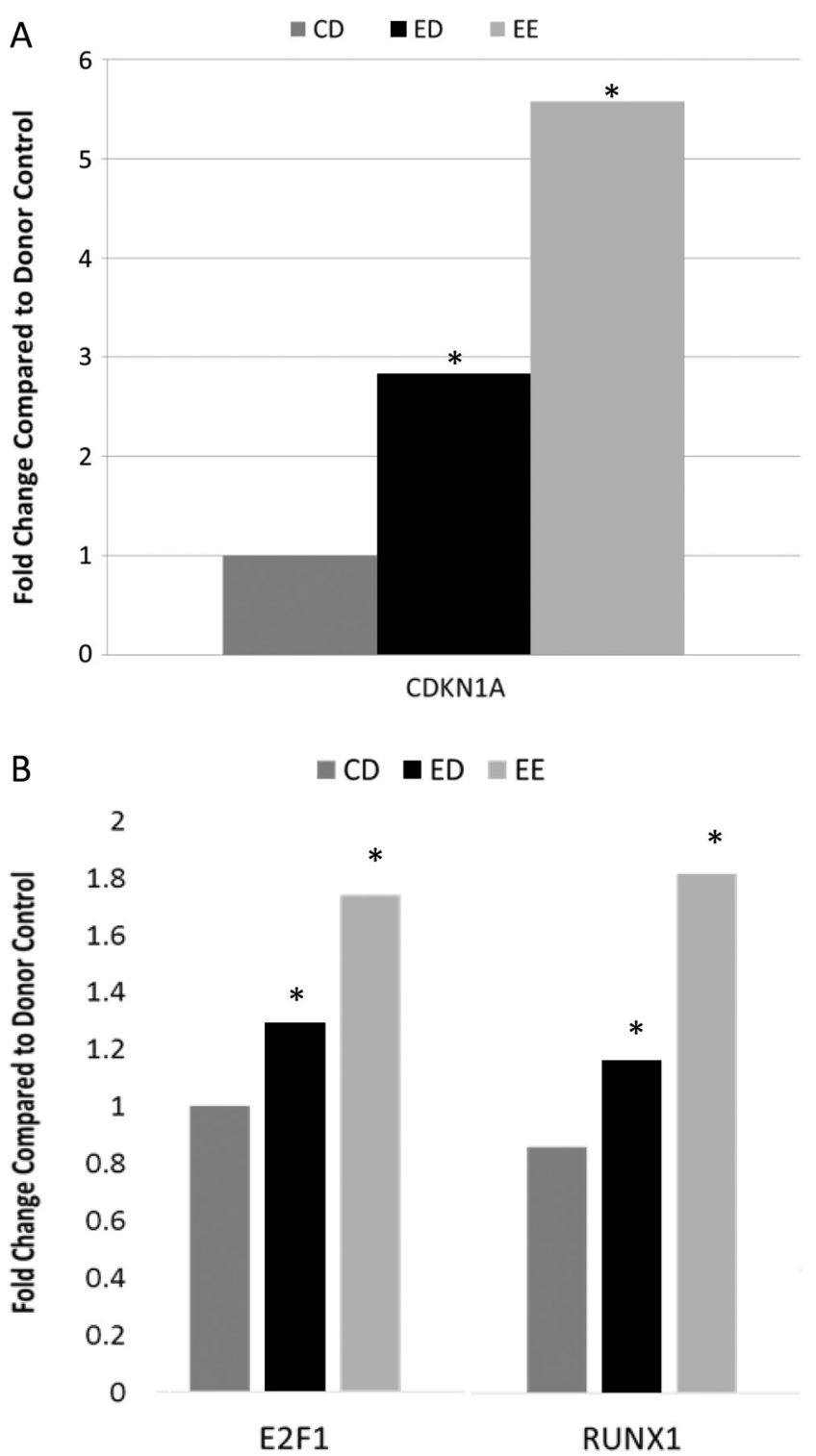

Figure 6 (A) miR-106a target gene validation of endometriosis derived blastocysts revealed significantly increased expression of CDKN1A when Co-cultured with endometriosis derived endometrial cells compared to fertile control $(\mathrm{CD}=$ fertile control endometrium/ fertile control blastocyst; $\mathrm{ED}=$ endometriosis endometrium/fertile control blastocyst; $\mathrm{EE}=$ endometriosis endometrium/endometriosis blastocyst) $\left({ }^{*} P \leq 0.05\right)$. (B) miR-106a target gene validation of endometriosis derived endometrial cells co-cultured with endometriosis derived blastocysts revealed significantly increased expression of E2F1 and RUNX1 compared to fertile control derived controls $(\mathrm{CD}=$ fertile control endometrium/fertile control blastocyst; $\mathrm{ED}=$ endometriosis endometrium/fertile control blastocyst; $\mathrm{EE}=$ endometriosis endometrium/endometriosis blastocyst) $(* P \leq 0.05)$.

delicate balance, and if the immune response is too great or insufficient, implantation potential may be impacted (Clark 2008). miR-150 is also a regulatory miRNA for the platelet-derived growth factor/VEGF family of genes, responsible for both angiogenesis and vasculogenesis, 
and is vital for successful embryo implantation. It has also been shown that supplementing embryo culture media with VEFG had beneficial effects with regard to postcompaction mouse embryo development, outgrowth, implantation and fetal development (Binder et al. 2014, 2016).

VEGFA is a glycosylated mitogen that specifically acts on endothelial cells with various effects, including mediating vascular permeability, angiogenesis, cell growth and cell migration and inhibiting apoptosis. Treatment of human endometrial cells with recombinant human VEGFA protein has been found to significantly increase endometrial cell adhesion (Hannan et al. 2011). In this study, VEGFA displayed a significantly lower expression in AMA samples $(P \leq 0.05)$, which may lead to dysregulated cellular function and increased apoptosis. This decrease in VEGFA expression may be indicative of the compromised communication between the AMA blastocyst and fertile control endometrium.

An additional altered miRNA observed in extracellular vesicles following co-culture was miR135a with an 8-fold decrease in expression in AMA blastocysts $(P \leq 0.05)$. A previous study observed that increased expression of miR-135a during preimplantation embryo development led to the downregulation of E3 ubiquitin ligase seven in absentia homologue 1A (SIAH1A) expression (Pang et al. 2011). The SIAH1A protein is involved in many cellular processes including apoptosis, TNF-alpha signaling and cell cycle regulation (Relaix et al. 2000). Nearly a third of the zygotes injected with miR-135a inhibitor were observed to arrest in development. The mechanism for this disruption is believed to be due to dysregulated proteosomal degradation, possibly controlling the expression of chemokines in DNA-binding protein (Pang et al. 2011). miR-135a has also been correlated to increased apoptosis resistance in cancer cells by $B C L 2$ regulation, a protein which determines cell survival and death through the mitochondrial apoptotic signaling pathway. Dysregulation of BCL2 and disruption of the apoptotic pathway resulted in interference of not only the establishment of pregnancy, but also pregnancy maintenance if successful implantation was able to occur at all (Mathew et al. 2009, Pan et al. 2014). The dysregulation of miR-135a observed in our samples suggests a decline in the cellular regulation of blastocysts derived from AMA patients, thus decreasing their viability and implantation potential.

Another miRNA found in the extracellular vesicles of the supernatant that displayed significantly disrupted differential expression was miR-449b. One strong, evidence-based target gene for miR-449b is NOTCH1 which has demonstrated its ability to act as a promoter of centriole multiplication multiciliogenesis by repressing the Delta/Notch pathway in Xenopus laevis embryonic dermis (Marcet et al. 2011). It is currently unknown precisely how mammalian cells are affected by this pathway; however it is hypothesized that, rather than the cilia found in Xenopus cells, it is the formation of the pseudopodia within the blastocyst trophectoderm that is being directly regulated (Goossens et al. 2013). miR-449b showed significantly increased expression in the co-culture supernatant of AMA blastocysts, which may indicate compromised trophectoderm formation, leading to an increase in failed implantation as observed clinically in this patient population.

\section{Endometriosis}

Endometriosis is a disease characterized by an abnormal, and in many cases, compromised endometrium. The impact this diagnosis bears on the bi-directional embryoendometrial dialogue at the time of implantation is largely unexplored. In our study, co-culture of endometriosis endometrium with blastocysts derived from patients diagnosed with endometriosis displayed significantly decreased extracellular miRNA, from within the isolated extracellular vesicles, when compared to fertile control endometrium with fertile control derived blastocysts for 10 key miRNAs $(P \leq 0.05)$. Of particular interest was miR-106a, commonly associated with adherens junctions, receptor interactions, metabolic pathways and VEGF signaling. In one study utilizing a uterine cavity lavage, miR-106a was identified in the exosomes found within the mucus near the time implantation may occur. It is suspected that as the blastocyst transitions toward the implantation site and begins to adhere, exosomes trapped within the mucus on the luminal surface of the endometrium release miRNAs which contribute to endometrial-embryo crosstalk by binding to the trophectoderm cells themselves $(\mathrm{Ng}$ et al. 2013). miR-106a has also revealed itself to be a strong identifying biomarker for various types of cancer where it is often down-regulated (Wang 2016). MiRTarBase target gene analysis of miR-106a identified 29 strong evidence-based target genes, including CDKN1A, E2F1 and RUNX1.

CDKN1A works in conjunction with TP53 and is linked to stress response in the G1 phase. If this stress response continues to cascade, it causes arrested cell cycle, potentially compromising the viability of embryos, and also contributing to possible de-regulation of the endometrium itself (Sharma et al. 2016). CDKN1A expression has been shown to induce cellular growth, arrest, terminal differentiation or apoptosis (Ying et al. 2011). Altered aberrant CDKN1A expression is also believed to play a vital role in the pathogenesis of specific cancer types, including ovarian and uterine carcinomas (Elbendary et al. 1996). Our results demonstrate altered CDKN1A gene expression in blastocysts derived from patients diagnosed with endometriosis, which may negatively impact their viability, and also contribute to the reduced implantation potential of embryos derived from this patient population. 
E2F1 is a transcription factor that plays a crucial role in the control of the cell cycle and can mediate apoptosis. Over-expression of E2F1 in myoblasts has demonstrated proliferation stimulation whilealso inhibiting differentiation (Luo et al. 2016). E2F1 induces the transcription of several genes involved in cell cycle entry, resulting in either the induction or inhibition of apoptosis. This influence leads to both negative and positive feedback loops, having a critical impact on the outcome of the cell's fate. The E2F1 protein has also shown increased expression in patients diagnosed with endometrial cancer, where the cell cycle becomes accelerated and irregular (Mints et al. 2016). Decreased expression of miR-106a can increase p53 expression in human glioma cells by inhibiting E2F1, negatively effecting cell proliferation and inducing apoptosis (Yang et al. 2011, Feng et al. 2013). Within the endometrium derived from patients diagnosed with endometriosis, irregular feedback loops may lead to inhibited differentiation, compromising the viability of the implantation site.

$R U N X 1$ is also a transcription factor that, when over expressed, increases apoptosis. In a mouse embryo knockout study, embryos with homozygous mutations on Runx 1 terminated after 12.5 days of development, and suffered from a lack of fetal liver hematopoiesis as well as hemorrhaging of the central nervous system (Okuda et al. 1996, Wang et al. 1996). Similar to E2F1, over-expression of the RUNX1 protein has been linked to various forms of cancer, including endometrial and endometrioid carcinomas (Planaguma et al. 2004, Abal et al. 2006). In mice, Runx1 also plays an important role in regulating the gene expression of Ada in trophoblast cell lineage which is critical for embryo development (Schaubach et al. 2006). In our study, endometriosis derived endometrium showed increased expression of RUNX1 that may lead to decreased implantation potential by compromising hematopoiesis and influencing the gene expression of placental $A D A$.

DAVID functional annotation bioinformatics microarray analysis revealed further dysfunction within the expression of miRNAs for both infertility etiologies, in relation to GO biological processes and pathways. When comparing the three different design groups, we found that miR-24, 34b and 484 were not commonly expressed between the groups of ED vs $C D$ and $E E$ vs $C D$, highlighting a subset of developmental pathways that were affected by the presence of endometrial cells from patients diagnosed with endometriosis (Supplementary Table 3). Interestingly, there were also no significant differences in miRNA expression between the groups of $E E$ and $E D$ $(P>0.05)$, which may signify that the endometriosis EEC are contributing the majority of the extracellular vesicles to the supernatant and may impact the developmental potential of the implanting blastocyst. One commonality between the endometriosis and AMA groups was the focus on cell cycle regulation. This may be indicative of compromised dialogue within the extracellular matrix. As the window of implantation approaches, specific auto-induction loops must occur to mediate the critical crosstalk between a competent blastocyst and the uterine epithelium at the implantation site (Hamatani et al. 2004). If these critical processes and pathways are disrupted, the resulting cell cycle dysregulation may contribute to a harsh environment, unsuitable for implantation and further development necessary for a viable pregnancy by preventing firm adhesion between the blastocyst and the uterine epithelium (Wang et al. 2008).

A few limitations of this study include the source of the endometrial biopsy. These biopsies were taken at the time of oocyte retrieval from women undergoing controlled ovarian stimulation. It has been shown that this leads to estrogen-dependent changes in the protein profiles of the endometrial compartments which may affect endometrial receptivity (Ullah et al. 2017). Furthermore, due to the scarcity of research materials, these protein profile alterations were unable to be validated further by ELISA analysis.

In conclusion, our study displayed a theme of compromised cellular communication in blastocysts and endometrium derived from patients diagnosed with AMA or endometriosis. These alterations may account for the lower implantation potential and clinical outcomes observed for these infertile patient populations. Any compromise in the delicate embryoendometrial molecular dialogue, either autocrine or paracrine, may impact transcription levels of key miRNAs and their target signaling molecules, resulting in significantly lower potential for reproductive success. Further investigation of the bi-directional molecular dialogue may help elucidate important implantation biomarkers during this critical time point, and advance our understanding of reproductive success.

\section{Supplementary data}

This is linked to the online version of the paper at https://doi.org/10.1530/REP-17-0566.

\section{Declaration of interest}

The authors declare that there is no conflict of interest that could be perceived as prejudicing the impartiality of the research reported.

\section{Funding}

This research did not receive any specific grant from any funding agency in the public, commercial or not-for-profit sector.

\section{Acknowledgements}

The authors would like to acknowledge the physicians, embryologists and surgical staff at the Colorado Center for Reproductive Medicine for their assistance with subject 
recruitment and sample collection. The authors would also like to thank Professor Carlos Simón and Fundación IVI for sharing their co-culture experimental protocols.

\section{References}

Abal M, Planaguma J, Gil-Moreno A, Monge M, Gonzalez M, Baro T, Garcia A, Castellvi J, Ramon YCS, Xercavins J et al. 2006 Molecular pathology of endometrial carcinoma: transcriptional signature in endometrioid tumors. Histology and Histopathology 21 197-204. (https://doi.org/10.14670/HH-21.197)

Altmae S, Martinez-Conejero JA, Esteban FJ, Ruiz-Alonso M, Stavreus-Evers A, Horcajadas JA \& Salumets A 2013 MicroRNAs miR-30b, miR-30d, and miR-494 regulate human endometrial receptivity. Reproductive Sciences 20 308-317. (https://doi. org/10.1177/1933719112453507)

Barmat LI, Liu HC, Spandorfer SD, Kowalik A, Mele C, Xu K, Veeck L, Damario M \& Rosenwaks Z 1999 Autologous endometrial co-culture in patients with repeated failures of implantation after in vitro fertilizationembryo transfer. Journal of Assisted Reproduction and Genetics 16 121-127. (https://doi.org/10.1023/A:1022575630105)

Binder NK, Evans J, Gardner DK, Salamonsen LA \& Hannan NJ 2014 Endometrial signals improve embryo outcome: functional role of vascular endothelial growth factor isoforms on embryo development and implantation in mice. Human Reproduction 29 2278-2286. (https://doi. org/10.1093/humrep/deu211)

Binder NK, Evans J, Salamonsen LA, Gardner DK, Kaitu'u-Lino TJ \& Hannan NJ 2016 Placental growth factor is secreted by the human endometrium and has potential important functions during embryo development and implantation. PLOS ONE 11 e0163096. (https://doi. org/10.1371/journal.pone.0163096)

Blakaj A \& Lin H 2008 Piecing together the mosaic of early mammalian development through microRNAs. Journal of Biological Chemistry 283 9505-9508. (https://doi.org/10.1074/jbc.R800002200)

Buck Louis GM, Hediger ML, Peterson CM, Croughan M, Sundaram R, Stanford J, Chen Z, Fujimoto VY, Varner MW, Trumble A et al. 2011 Incidence of endometriosis by study population and diagnostic method: the ENDO study. Fertility and Sterility 96 360-365. (https://doi. org/10.1016/j.fertnstert.2011.05.087)

Clark DA 2008 Immunological factors in pregnancy wastage: fact or fiction. American Journal of Reproductive Immunology 59 277-300. (https://doi. org/10.1111/j.1600-0897.2008.00580.x)

Das SK 2009 Cell cycle regulatory control for uterine stromal cell decidualization in implantation. Reproduction 137 889-899. (https:// doi.org/10.1530/REP-08-0539)

Elbendary AA, Cirisano FD, Evans AC Jr, Davis PL, Iglehart JD, Marks JR \& Berchuck A 1996 Relationship between p21 expression and mutation of the p53 tumor suppressor gene in normal and malignant ovarian epithelial cells. Clinical Cancer Research 2 1571-1575.

Elton TS, Selemon H, Elton SM \& Parinandi NL 2013 Regulation of the MIR155 host gene in physiological and pathological processes. Gene 532 1-12. (https://doi.org/10.1016/j.gene.2012.12.009)

Feng S, Cao Z \& Wang X 2013 Role of aryl hydrocarbon receptor in cancer. Biochimica et Biophysica Acta 1836 197-210. (https://doi.org/10.1016/j. bbcan.2013.05.001)

Garcia-Velasco JA \& Quea G 2005 Medical treatment of endometriosis. Minerva Ginecologica 57 249-255.

Gardner DK \& Schoolcraft WB 1999. In vitro culture of human blastocysts. In Towards Reproductive Certainty: Fertility and Genetics Beyond., pp 378-388.Carnforth: Parthenon Press

Goossens K, Mestdagh P, Lefever S, Van Poucke M, Van Zeveren A, Van Soom A, Vandesompele J \& Peelman L 2013 Regulatory microRNA network identification in bovine blastocyst development. Stem Cells and Development 22 1907-1920. (https://doi.org/10.1089/scd.2012.0708)

Hamatani T, Daikoku T, Wang H, Matsumoto H, Carter MG, Ko MS \& Dey SK 2004 Global gene expression analysis identifies molecular pathways distinguishing blastocyst dormancy and activation. PNAS 101 10326-10331. (https://doi.org/10.1073/pnas.0402597101)

Hannan NJ, Paiva P, Meehan KL, Rombauts LJ, Gardner DK \& Salamonsen LA 2011 Analysis of fertility-related soluble mediators in human uterine fluid identifies VEGF as a key regulator of embryo implantation. Endocrinology 152 4948-4956. (https://doi.org/10.1210/ en.2011-1248)

Hannan NJ, Nie G, Rainzcuk A, Rombauts LJ \& Salamonsen LA 2012 Uterine lavage or aspirate: which view of the intrauterine environment? Reproductive Sciences 19 1125-1132. (https://doi. org/10.1177/1933719112443879)

Harton GL, Munne S, Surrey M, Grifo J, Kaplan B, McCulloh DH, Griffin DK, Wells D \& PGD Practitioners Group 2013 Diminished effect of maternal age on implantation after preimplantation genetic diagnosis with array comparative genomic hybridization. Fertility and Sterility $\mathbf{1 0 0}$ 1695-1703. (https://doi.org/10.1016/j.fertnstert.2013.07.2002)

Kovacic B 2012 Culture systems: low-oxygen culture. Methods in Molecular Biology 912 249-272.

Kuwayama M, Vajta G, Kato O \& Leibo SP 2005 Highly efficient vitrification method for cryopreservation of human oocytes. Reproductive BioMedicine Online 11 300-308. (https://doi.org/10.1016/S1472-6483(10)60837-1)

Laurent LC 2008 MicroRNAs in embryonic stem cells and early embryonic development. Journal of Cellular and Molecular Medicine 12 2181-2188. (https://doi.org/10.1111/j.1582-4934.2008.00513.x)

Luo W, Li G, Yi Z, Nie Q \& Zhang X 2016 E2F1-miR-20a-5p/20b-5p auto-regulatory feedback loop involved in myoblast proliferation and differentiation. Scientific Reports 6 27904. (https://doi.org/10.1038/ srep27904)

Marcet B, Chevalier B, Luxardi G, Coraux C, Zaragosi LE, Cibois M, RobbeSermesant K, Jolly T, Cardinaud B, Moreilhon C et al. 2011 Control of vertebrate multiciliogenesis by miR-449 through direct repression of the Delta/Notch pathway. Nature Cell Biology 13 693-699. (https://doi. org/10.1038/ncb2241)

Mathew SJ, Haubert D, Kronke M \& Leptin M 2009 Looking beyond death: a morphogenetic role for the TNF signalling pathway. Journal of Cell Science 122 1939-1946. (https://doi.org/10.1242/jcs.044487)

Mercader A, Garcia-Velasco JA, Escudero E, Remohi J, Pellicer A \& Simon C 2003 Clinical experience and perinatal outcome of blastocyst transfer after coculture of human embryos with human endometrial epithelial cells: a 5-year follow-up study. Fertility and Sterility $\mathbf{8 0}$ 1162-1168. (https://doi.org/10.1016/S0015-0282(03)01178-6)

Mints M, Mushtaq M, lurchenko N, Kovalevska L, Stip MC, Budnikova D, Andersson S, Polischuk L, Buchynska L \& Kashuba E 2016 Mitochondrial ribosomal protein $\mathrm{S} 18-2$ is highly expressed in endometrial cancers along with free E2F1. Oncotarget 7 22150-22158. (https://doi.org/10.18632/ oncotarget.7905)

$\mathrm{Ng} \mathrm{YH}$, Rome S, Jalabert A, Forterre A, Singh H, Hincks CL \& Salamonsen LA 2013 Endometrial exosomes/microvesicles in the uterine microenvironment: a new paradigm for embryo-endometrial cross talk at implantation. PLoS ONE 8 e58502. (https://doi.org/10.1371/journal.pone.0058502)

Ocal P, Cift T, Bulut B, Balcan E, Cepni I, Aydogan B \& Irez T 2012 Recurrent implantation failure is more frequently seen in female patients with poor prognosis. International Journal of Fertility and Sterility $\mathbf{6}$ 71-78.

Okuda T, van Deursen J, Hiebert SW, Grosveld G \& Downing JR 1996 AML1, the target of multiple chromosomal translocations in human leukemia, is essential for normal fetal liver hematopoiesis. Cell $\mathbf{8 4}$ 321-330. (https://doi.org/10.1016/S0092-8674(00)80986-1)

Ozkan S, Murk W \&Arici A 2008 Endometriosis and infertility: epidemiology and evidence-based treatments. Annals of the New York Academy of Sciences 1127 92-100. (https://doi.org/10.1196/annals.1434.007)

Pan Q \& Chegini N 2008 MicroRNA signature and regulatory functions in the endometrium during normal and disease states. Seminars in Reproductive Medicine 26 479-493. (https://doi.org/10.1055/s-0028-1096128)

Pan Y, Ren F, Zhang W, Liu G, Yang D, Hu J, Feng K \& Feng Y 2014 Regulation of BGC-823 cell sensitivity to adriamycin via miRNA-135a-5p. Oncology Reports 32 2549-2556. (https://doi.org/10.3892/or.2014.3546)

Pang RT, Liu WM, Leung CO, Ye TM, Kwan PC, Lee KF \& Yeung WS 2011 miR-135A regulates preimplantation embryo development through down-regulation of E3 Ubiquitin Ligase Seven In Absentia Homolog 1A (SIAH1A) expression. PLoS ONE 6 e27878. (https://doi.org/10.1371/ journal.pone.0027878)

Planaguma J, Diaz-Fuertes M, Gil-Moreno A, Abal M, Monge M, Garcia A, Baro T, Thomson TM, Xercavins J, Alameda F et al. 2004 A differential gene expression profile reveals overexpression of RUNX1/AML1 in invasive endometrioid carcinoma. Cancer Research 64 8846-8853. (https://doi.org/10.1158/0008-5472.CAN-04-2066) 
Practice Committee of the American Society for Reproductive Medicine 2006 Endometriosis and infertility. Fertility and Sterility 86 S156-S160.

Relaix F, Wei X, Li W, Pan J, Lin Y, Bowtell DD, Sassoon DA \& Wu X 2000 Pw1/Peg3 is a potential cell death mediator and cooperates with Siah1a in p53-mediated apoptosis. PNAS 97 2105-2110. (https://doi. org/10.1073/pnas.040378897)

Rizk B, Turki R, Lotfy H, Ranganathan S, Zahed H, Freeman AR, Shilbayeh Z, Sassy M, Shalaby M \& Malik R 2015 Surgery for endometriosis-associated infertility: do we exaggerate the magnitude of effect? Facts, Views and Vision in ObGyn 7 109-118.

Rosenwaks Z, Davis OK \& Damario MA 1995 The role of maternal age in assisted reproduction. Human Reproduction 10 (Supplement 1) 165-173. (https://doi.org/10.1093/humrep/10.suppl_1.165)

Rubio C, Simon C, Mercader A, Garcia-Velasco J, Remohi J \& Pellicer A 2000 Clinical experience employing co-culture of human embryos with autologous human endometrial epithelial cells. Human Reproduction 15 (Supplement 6) 31-38. (https://doi.org/10.1093/humrep/15.suppl_4.31)

Ruiz-Alonso M, Blesa D \& Simon C 2012 The genomics of the human endometrium. Biochimica et Biophysica Acta 1822 1931-1942. (https:// doi.org/10.1016/j.bbadis.2012.05.004)

Schaubach BM, Wen HY \& Kellems RE 2006 Regulation of murine Ada gene expression in the placenta by transcription factor RUNX1. Placenta 27 269-277. (https://doi.org/10.1016/j.placenta.2005.03.001)

Schoolcraft WB, Treff NR, Stevens JM, Ferry K, Katz-Jaffe M \& Scott RT Jr 2011 Live birth outcome with trophectoderm biopsy, blastocyst vitrification, and single-nucleotide polymorphism microarray-based comprehensive chromosome screening in infertile patients. Fertility and Sterility 96 638-640. (https://doi.org/10.1016/j.fertnstert.2011.06.049)

Shapiro BS, Daneshmand ST, Desai J, Garner FC, Aguirre M \& Hudson C 2016 The risk of embryo-endometrium asynchrony increases with maternal age after ovarian stimulation and IVF. Reproductive BioMedicine Online 33 50-55. (https://doi.org/10.1016/j.rbmo.2016.04.008)

Sharma N, Kubaczka C, Kaiser S, Nettersheim D, Mughal SS, Riesenberg S, Holzel M, Winterhager E \& Schorle H 2016 Tpbpa-Cre-mediated deletion of TFAP2C leads to deregulation of Cdkn1a, Akt1 and the ERK pathway, causing placental growth arrest. Development 143 787-798. (https://doi.org/10.1242/dev.128553)

Shebl O, Sifferlinger I, Habelsberger A, Oppelt P, Mayer RB, Petek E \& Ebner T 2017 Oocyte competence in in vitro fertilization and intracytoplasmic sperm injection patients suffering from endometriosis and its possible association with subsequent treatment outcome: a matched case-control study. Acta Obstetricia et Gynecologica Scandinavica 96 736-744. (https://doi.org/10.1111/aogs.12941)

Simon C, Moreno C, Remohi J \& Pellicer A 1998 Molecular interactions between embryo and uterus in the adhesion phase of human implantation. Human Reproduction 13 (Supplement 3) 219-232; discussion 233-216. (https://doi.org/10.1093/humrep/13.suppl_3.219)

Simon C, Mercader A, Garcia-Velasco J, Nikas G, Moreno C, Remohi J \& Pellicer A 1999 Coculture of human embryos with autologous human endometrial epithelial cells in patients with implantation failure. Journal of Clinical Endocrinology and Metabolism 84 2638-2646. (https://doi. org/10.1210/jcem.84.8.5873)

Tapia A, Vilos C, Marin JC, Croxatto HB \& Devoto L 2011 Bioinformatic detection of E47, E2F1 and SREBP1 transcription factors as potential regulators of genes associated to acquisition of endometrial receptivity. Reproductive Biology and Endocrinology 9 14. (https://doi. org/10.1186/1477-7827-9-14)
Tapia-Pizarro A, Figueroa P, Brito J, Marin JC, Munroe DJ \& Croxatto HB 2014 Endometrial gene expression reveals compromised progesterone signaling in women refractory to embryo implantation. Reproductive Biology and Endocrinology 12 92. (https://doi.org/10.1186/1477-782712-92)

Thoma ME, McLain AC, Louis JF, King RB, Trumble AC, Sundaram R \& Buck Louis GM 2013 Prevalence of infertility in the United States as estimated by the current duration approach and a traditional constructed approach. Fertility and Sterility 99 1324.e1321-1331.e1321. (https://doi. org/10.1016/j.fertnstert.2012.11.037)

Ullah K, Rahman TU, Pan HT, Guo MX, Dong XY, Liu J, Jin LY, Cheng Y, Ke ZH, Ren J et al. 2017 Serum estradiol levels in controlled ovarian stimulation directly affect the endometrium. Journal of Molecular Endocrinology 59 105-119. (https://doi.org/10.1530/JME-17-0036)

Wang H 2016 Predicting microRNA biomarkers for cancer using phylogenetic tree and microarray analysis. International Journal of Molecular Sciences 17 E773. (https://doi.org/10.3390/ijms17122139)

Wang Q, Stacy T, Binder M, Marin-Padilla M, Sharpe AH \& Speck NA 1996 Disruption of the Cbfa2 gene causes necrosis and hemorrhaging in the central nervous system and blocks definitive hematopoiesis. PNAS 93 3444-3449. (https://doi.org/10.1073/pnas.93.8.3444)

Wang B, Sheng JZ, He RH, Qian YL, Jin F \& Huang HF 2008 High expression of L-selectin ligand in secretory endometrium is associated with better endometrial receptivity and facilitates embryo implantation in human being. American Journal of Reproductive Immunology $\mathbf{6 0}$ 127-134. (https://doi.org/10.1111/j.1600-0897.2008.00604.x)

Weimar CH, Post Uiterweer ED, Teklenburg G, Heijnen CJ \& Macklon NS 2013 In-vitro model systems for the study of human embryo-endometrium interactions. Reproductive BioMedicine Online 27 461-476. (https://doi. org/10.1016/j.rbmo.2013.08.002)

Wenzl R, Kiesel L, Huber JC \& Wieser F 2003 Endometriosis: a genetic disease. Drugs Today 39 961-972. (https://doi.org/10.1358/ dot.2003.39.12.799414)

Wilcox AJ, Baird DD \& Weinberg CR 1999 Time of implantation of the conceptus and loss of pregnancy. New England Journal of Medicine 340 1796-1799. (https://doi.org/10.1056/NEJM199906103402304)

Yang G, Zhang R, Chen X, Mu Y, Ai J, Shi C, Liu Y, Shi C, Sun L, Rainov NG et al. 2011 MiR-106a inhibits glioma cell growth by targeting E2F1 independent of p53 status. Journal of Molecular Medicine 89 1037-1050. (https://doi.org/10.1007/s00109-011-0775-x)

Ying TH, Tseng CJ, Tsai SJ, Hsieh SC, Lee HZ, Hsieh YH \& Bau DT 2011 Association of p53 and CDKN1A genotypes with endometriosis. Anticancer Research 31 4301-4306.

Zhang J 2015 Revisiting germinal vesicle transfer as a treatment for aneuploidy in infertile women with diminished ovarian reserve. Journal of Assisted Reproduction and Genetics 32 313-317. (https://doi. org/10.1007/s10815-014-0400-3)

Received 12 September 2017

First decision 12 October 2017

Revised manuscript received 4 April 2018

Accepted 10 April 2018 